\title{
THE ISOMORPHISM RELATION FOR SEPARABLE C*-ALGEBRAS
}

\author{
George A. Elliott, Ilijas Farah, Vern I. Paulsen, Christian Rosendal, \\ Andrew S. TOMS AND Asger TÖRnquist
}

\begin{abstract}
We prove that the isomorphism relation for separable $\mathrm{C}^{*}$-algebras, the relations of complete and $n$-isometry for operator spaces, and the relations of unital $n$-order isomorphisms of operator systems, are Borel reducible to the orbit equivalence relation of a Polish group action on a standard Borel space.
\end{abstract}

\section{Introduction}

The problem of classifying a collection of objects up to some notion of isomorphism can usually be couched as the study of an analytic equivalence relation on a standard Borel space parametrizing the objects in question. Such relations admit a notion of comparison, Borel reducibility, which allows one to assign a degree of complexity to the classification problem. If $X$ and $Y$ are standard Borel spaces admitting equivalence relations $E$ and $F$ respectively then we say that $E$ is Borel reducible to $F$, written $E \leq_{\mathrm{B}} F$, if there is a Borel map $\Theta: X \rightarrow Y$ such that

$$
x E y \Longleftrightarrow \Theta(x) F \Theta(y) \text {. }
$$

In other words, $\Theta$ carries equivalence classes to equivalence classes injectively. We view $E$ as being "less complicated" than $F$. There are some particularly prominent degrees of complexity in this theory which serve as benchmarks for classification problems in general. For instance, a relation $E$ is classifiable by countable structures (CCS) if it is Borel reducible to the isomorphism relation for countable graphs. Classification problems in functional analysis (our interest here) tend not to be CCS, but may nevertheless be "not too complicated" in that they are Borel reducible to the orbit equivalence relation of a Borel action of a Polish group on a standard Borel space; this property is known as being below a group action.

The connections between Borel reducibility and operator algebras have received considerable attention lately. Using Hjorth's theory of turbulence developed in [10], it has been shown that several classes of operator algebras are not CCS. This applies to von Neumann factors of every type [18], ITPFI ${ }_{2}$ factors [19], simple unital nuclear $\mathrm{C}^{*}$-algebras [7], and the spaces of irreducible representations of non-type $\mathrm{I}^{*}$-algebras $[4,13]$. On the other hand, Elliott's $K$-theoretic classification of AF algebras together with the Borel computability of $K$-theory [6] show that AF algebras are CCS (see, e.g., $[2,3])$.

We are interested in classifying separable $\mathrm{C}^{*}$-algebras, operator spaces, and operator systems. The classification problem for nuclear simple separable $\mathrm{C}^{*}$-algebras

Received by the editors June 28, 2013. 
was studied in [7], where the isomorphism relation was shown to be below a group action. Establishing this upper bound was rather involved; it required Borel versions of Kirchberg's $\mathcal{O}_{2}$ embedding and absorption theorems. It of course invited the question of whether isomorphism of all separable $\mathrm{C}^{*}$-algebras is below a group action. Here we give a surprisingly simple proof of the following result.

Theorem 1.1. Each of the following equivalence relations is below a group action:

(1) The isomorphism relation for separable $C^{*}$-algebras.

(2) Complete isometry, as well as n-isometry for any $n$, of separable operator spaces.

(3) Complete isometry, as well as n-isometry for any n, of separable operator systems.

(4) Unital complete order isomorphism, as well as unital n-order isomorphism for any $n$, of separable unital operator systems.

Part (1) of course improves on one of the main results of [7]. Parts (2-4) give a partial answer to a question raised by Ed Effros during the January 2012 meeting "Set theory and $C^{*}$-algebras" at the American Institute of Mathematics. Theorem 1.1 follows from a more general result proved in Section 2. We show that Iso(U), the isometry group of the Urysohn metric space $(\mathbb{U}, \delta)$, plays a role for the isometry relation of what we term Polish structures in a countable signature (see $[1,5]$ ) analogous to the role played by the Polish group $S_{\infty}$ of all permutations of $\mathbb{N}$ for the isomorphism relation of countable structures.

\section{Polish structures}

The main ingredients for proving Theorem 1.1 are the notion of a Polish structure, which we introduce now, and Theorem 2.2 below.

Definition 2.1. Let $\mathcal{L}=\left(l_{1}, \ldots\right)$ be a finite or infinite sequence in $\mathbb{N}$. A Polish $\mathcal{L}$-structure ${ }^{1}$ is a triple

$$
\mathbb{X}=\left(X, d^{\mathbb{X}},\left(F_{n}^{\mathbb{X}}\right)\right),
$$

where $\left(X, d^{\mathbb{X}}\right)$ is a separable complete metric space, called the domain, and $F_{n}^{\mathbb{X}} \subseteq X^{l_{n}}$ is closed in the product topology. We think of $F_{n}^{\mathbb{X}}$ as a relation on $X$ and call $l_{n}$ the arity of $F_{n}^{\mathbb{X}}$. The sequence $\mathcal{L}$ is called the signature or arity sequence of the structure $\mathbb{X}$. (Below we will suppress the superscript $\mathbb{X}$ whenever possible.)

Two Polish structures $\mathbb{X}$ and $\mathbb{Y}$ with the same signature $\mathcal{L}$ are said to be isometrically isomorphic if there is an isometric bijection $h: X \rightarrow Y$ such that for all $n$ and $\left(x_{1}, \ldots, x_{l_{n}}\right) \in X^{l_{n}}$ we have

$$
\left(x_{1}, \ldots, x_{l_{n}}\right) \in F_{n}^{\mathbb{X}} \Longleftrightarrow\left(h\left(x_{1}\right), \ldots, h\left(x_{l_{n}}\right)\right) \in F_{n}^{\mathbb{Y}} .
$$

Let $(Y, d)$ be a metric space, let $\mathcal{L}=\left(l_{1}, \ldots\right)$ be given, and define $M(\mathcal{L}, Y, d) \subseteq$ $(F(Y) \backslash\{\varnothing\}) \times \prod_{n} F\left(Y^{l_{n}}\right)$ by

$$
M(\mathcal{L}, Y, d)=\left\{\left(X,\left(F_{n}\right)\right): F_{n} \subseteq X^{l_{n}}\right\} .
$$

This set is Borel (by $[12,12.11])$, and to each $\mathbb{X}=\left(X,\left(F_{n}\right)\right) \in M(\mathcal{L}, Y, d)$ we have the Polish $\mathcal{L}$-structure $\left(X, d \uparrow X,\left(F_{n}\right)\right)$, which we also denote by $\mathbb{X}$. For $\mathbb{X}, \mathbb{Y} \in M(\mathcal{L}, Y, d)$, write $\mathbb{X} \simeq^{M(\mathcal{L}, Y, d)} \mathbb{Y}$ if $\mathbb{X}$ and $\mathbb{Y}$ are isomorphic Polish $\mathcal{L}$-structures.

\footnotetext{
${ }^{1}$ We consciously avoid the term "metric structure" since this is already used for a slightly different notion in continuous logic.
} 
A metric structure as defined in [1] can also involve predicates, i.e., certain continuous functions $f$ from $X^{n}$ for some $n$ into $[0,1]$. For our purposes, there is no need to extend the definition to accommodate such predicates since such a function is coded by the closed sets $\left\{\bar{x} \in X^{n}: f(\bar{x}) \geq q\right\}$ for $q \in \mathbb{Q}$.

When $(Y, d)$ is the Urysohn metric space $\mathbb{U}$, we will usually write $M(\mathcal{L})$ rather than $M(\mathcal{L}, \mathbb{U}, \delta)$. This is motivated by the fact that every separable metric space can be isometrically embedded into $\mathbb{U}$, and so every Polish $\mathcal{L}$-structure is isomorphic to some $\mathbb{X} \in M(\mathcal{L})$. Thus, $M(\mathcal{L})$ provides a parametrization of all Polish $\mathcal{L}$-structures as a standard Borel space. It is not hard to see that that this moreover is a good standard Borel parametrization in the sense of [7].

The group Iso(U) acts diagonally on $\mathbb{U}^{l_{i}}$ for each $i$, and so it acts naturally on $M(\mathcal{L})$ by $\sigma \cdot\left(X,\left(F_{n}\right)\right)=\left(\sigma \cdot X,\left(\sigma \cdot F_{n}\right)\right)$. This induces the orbit equivalence relation

$$
\mathcal{X} \equiv{ }^{M(\mathcal{L})} \mathcal{Y} \Longleftrightarrow(\exists \sigma \in \operatorname{Iso}(\mathbb{U})) \quad \sigma \cdot \mathcal{X}=\mathcal{Y} .
$$

The following theorem may be viewed as an elaboration of the results in [9, Section 2]. However, the explicit Borel computation of embeddings done in Lemma 2.3 below will be needed not just to prove the theorem, but also several times later.

Theorem 2.2. We have $\simeq^{M(\mathcal{L})} \leq_{\mathrm{B}} \equiv^{M(\mathcal{L})}$, and so $\simeq^{M(\mathcal{L})}$ is below a group action.

To prove this, we first need a uniformly Borel version of the injective universality and homogeneity of $\mathbb{U}$. For a countable set $A$, define

$$
\mathcal{M}_{A}=\left\{d \in \mathbb{R}^{A \times A}: d \text { is a metric on } A\right\} .
$$

This is easily seen to form a $G_{\delta}$ subset of $\mathbb{R}^{A \times A}$, when $\mathcal{M}_{A}$ is Polish in the subspace topology. When $A=\mathbb{N}$ we let $\mathcal{M}=\mathcal{M}_{\mathbb{N}}$.

Lemma 2.3. There are Borel functions $\vartheta_{n}: \mathcal{M} \rightarrow \mathbb{U}, n \in \mathbb{Z}$, such that for all $d, d^{\prime} \in \mathcal{M}$ :

(1) the set $\left\{\vartheta_{n}(d): n \in \mathbb{Z}\right\}$ is dense in $\mathbb{U}$;

(2) $\delta\left(\vartheta_{n}(d), \vartheta_{m}(d)\right)=d(n, m)$ for all $n, m \in \mathbb{N}$;

(3) any isometric bijection $\sigma: \overline{\left\{\vartheta_{n}(d): n \in \mathbb{N}\right\}} \rightarrow \overline{\left\{\vartheta_{n}\left(d^{\prime}\right): n \in \mathbb{N}\right\}}$ extends to an isometric automorphism of $\mathbb{U}$.

Proof. We adapt the Katetov construction of the Urysohn metric space; see $[9,11]$. Fix an enumeration $\left(A_{n}\right)_{n \in \mathbb{N}}$ of all finite non-empty subsets of $\mathbb{Z}$, and let $*$ be a point not in $\mathbb{Z}$. For $d \in \mathcal{M}$, call a metric $\rho \in \mathcal{M}_{\mathbb{Z}}$ a Katetov extension of $d$ if $\rho \uparrow \mathbb{N}=d$, and for all $n \in \mathbb{N}$, all $\varepsilon>0$, and every metric $\tilde{d}$ on $A_{n} \cup\{*\}$ satisfying

(1) $\rho(x, y)=\tilde{d}(x, y)$ for all $x, y \in A_{n}$, and

(2) $\tilde{d}(x, *) \in \mathbb{Q}_{+}$for all $x \in A_{n}$

there is some $i \in \mathbb{Z} \backslash \mathbb{N}$ such that $|\rho(i, x)-\tilde{d}(*, x)|<\varepsilon$ for all $x \in A_{n}$. It follows directly from the definition that the set

$$
U=\left\{(d, \rho) \in \mathcal{M} \times \mathcal{M}_{\mathbb{Z}}: \rho \text { is a Katetov extension of } d\right\}
$$

is a $G_{\delta}$. The group $\operatorname{Sym}(\mathbb{Z} \backslash \mathbb{N})$ of all permutations of $\mathbb{Z} \backslash \mathbb{N}$ acts naturally on $\mathcal{M}_{\mathbb{Z}}$, and the sections $U_{d}=\{\rho:(d, \rho) \in U\}$ are invariant under this action. The Katetov property guarantees that the $\operatorname{Sym}(\mathbb{Z} \backslash \mathbb{N})$-orbits in $U_{d}$ are dense in $U_{d}$. It follows from Theorem A (see appendix) that there is a Borel map $\psi: \mathcal{M} \rightarrow \mathcal{M}_{\mathbb{Z}}$ such that 
$(d, \psi(d)) \in U$ for all $d \in \mathcal{M}$. A standard approximate intertwining/back-and-forth argument shows that for any $(d, \rho) \in U$, the completion of $(\mathbb{Z}, \rho)$ is isometrically isomorphic to $(\mathbb{U}, \delta)$, and a Borel coding of this argument analogous to $[7$, Theorem 7.6] shows that there are Borel maps $\vartheta_{n}: \mathcal{M} \rightarrow \mathbb{U}, n \in \mathbb{Z}$, satisfying (1) and (2) above. Finally, another approximate intertwining/back-and-forth argument can be used to establish (3); further details may be found in [9].

Proof of Theorem 2.2. We will define a Borel function $M(\mathcal{L}) \rightarrow M(\mathcal{L}): \mathbb{X} \mapsto \mathbb{X}^{\prime}$ such that $\mathbb{X} \simeq^{M(\mathcal{L})} \mathbb{Y}$ if and only if $\mathbb{X}^{\prime} \equiv^{M(\mathcal{L})} \mathbb{Y}^{\prime}$, and $\mathbb{X} \simeq^{M(\mathcal{L})} \mathbb{X}^{\prime}$. For $\mathbb{X}, \mathbb{Y} \in M(\mathcal{L})$ with domains $X$ and $Y$ finite, the homogeneity of $\mathbb{U}$ immediately gives that $\mathbb{X} \simeq^{M(\mathcal{L})} \mathbb{Y}$ if and only if $\mathbb{X} \equiv{ }^{M(\mathcal{L})} \mathbb{Y}$, so here we may simply take $\mathbb{X}=\mathbb{X}^{\prime}$.

For the case when $\mathbb{X}$ has infinite domain, recall that by the Kuratowski-RyllNardzewski theorem we may find Borel functions $\psi_{n}^{k}: F\left(\mathbb{U}^{k}\right) \rightarrow \mathbb{U}^{k}$ such that $\left\{\psi_{n}^{k}(F)\right.$ : $n \in \mathbb{N}\}$ is dense in $F$ whenever $F \neq \varnothing$. From the $\psi_{n}^{k}$ we can define a sequence of Borel functions $\tilde{\psi}_{n}: F(\mathbb{U}) \rightarrow \mathbb{U}$ such that whenever $\mathbb{X}=\left(X,\left(F_{k}\right)\right) \in M(\mathcal{L})$ and $X$ is infinite, the sequence $\tilde{\psi}_{n}(X)$ gives an injective enumeration of a dense subset of $X$ and for all $k, \psi_{n}^{l_{k}}\left(F_{k}\right) \subseteq\left\{\tilde{\psi}_{n}(X): n \in \omega\right\}^{l_{k}}$. Define an element of $d_{X} \in \mathcal{M}$ by $d_{X}(n, m)=\delta\left(\tilde{\psi}_{n}(X), \tilde{\psi}_{m}(x)\right)$, and set $\gamma_{n}(X)=\vartheta_{n}\left(d_{X}\right), n \in \mathbb{Z}$, where the $\vartheta_{n}$ are provided by Lemma 2.3. Then the $\gamma_{n}$ are Borel, and if we define $\mathbb{X}^{\prime}=\left(X^{\prime}, F_{n}^{\prime}\right)$ by setting

$$
X^{\prime}=\operatorname{cl}\left(\left\{\gamma_{n}(X): n \in \mathbb{N}\right\}\right)
$$

and

$$
\begin{aligned}
F_{n}^{\prime}= & \operatorname{cl}\left(\left\{\left(\gamma_{i_{1}}(X), \ldots, \gamma_{i_{n}}(X)\right):\right.\right. \\
& \left.\left.\left(\exists\left(i_{j}\right)\right)\left(\tilde{\psi}_{i_{1}}(X), \ldots, \tilde{\psi}_{i_{l_{n}}}(X)\right) \in F_{n}\right\}\right),
\end{aligned}
$$

then $\mathbb{X}^{\prime} \simeq^{M(\mathcal{L})} \mathbb{X}$. That the map $\mathbb{X} \mapsto \mathbb{X}^{\prime}$ is Borel follows from the Kuratowski-RyllNardzewski theorem, and that $\mathbb{X} \simeq{ }^{M(\mathcal{L})} \mathbb{Y}$ precisely when $\mathbb{X}^{\prime} \equiv^{M(\mathcal{L})} \mathbb{Y}^{\prime}$ is immediate from Lemma 2.3.(3).

\section{Proof of Theorem 1.1}

3.1. $\mathrm{C}^{*}$-algebras. A $\mathrm{C}^{*}$-algebra $A$ can be cast as a Polish structure with domain $A$ as follows: we have the relations $F_{0}=\{0\} \subseteq A, F_{+}, F . \subseteq A^{3}$, which are the graphs of the functions $(a, b) \mapsto a+b$ and $(a, b) \mapsto a b$, respectively, $F_{*} \subseteq A^{2}$ which is the graph of the map $a \mapsto a^{*}$, and for each $q \in \mathbb{Q}(i)=\mathbb{Q}+i \mathbb{Q}$ the relations

$$
F_{q}=\left\{(a, b) \in A^{2}: b=q a\right\} .
$$

The signature is $\mathcal{L}_{\mathrm{C}^{*}}=(1,3,3,2,2, \ldots)$. It is easy to check directly that the set $M_{\mathrm{C}^{*}} \subseteq$ $M\left(\mathcal{L}_{\mathrm{C}^{*}}\right)$ which corresponds to $\mathrm{C}^{*}$-algebras is Borel. (See also Lemma 3.1 below, where a more general statement is proven.) Let $\simeq{ }^{M_{\mathrm{C}^{*}}}$ denote the isomorphism relation in $M_{\mathrm{C}^{*}}$. It can easily be shown that $M_{\mathrm{C}^{*}}$ provides a standard Borel parametrization of the class of separable $\mathrm{C}^{*}$-algebras, in the sense of [7, Definition 2.1]). Clearly, $\simeq{ }^{M_{\mathrm{C}^{*}}}$ is the restriction of $\simeq^{M\left(\mathcal{L}_{\mathrm{C}^{*}}\right)}$ to $M_{\mathrm{C}^{*}}$, and so by Theorem 2.2 above $\simeq^{M_{\mathrm{C}^{*}}}$ is below a group action.

To finish the proof of Theorem 1.1.(1) we need only show that the parametrization $M_{\mathrm{C}^{*}}$ is weakly equivalent to the parametrizations given in [7], in the sense of $[7$, 
Definition 2.1]. For this, recall from [7, Section 2.4] that $\hat{\Xi}$ is the space of countable normed $\mathbb{Q}(i)$-*-algebras with domain $\mathbb{N}$ which satisfy the $\mathrm{C}^{*}$-axiom. For each $\xi \in$ $\hat{\Xi}$, let $\mathrm{C}^{*}(\xi)$ denote the $\mathrm{C}^{*}$-algebra obtained from completing $\xi$ and extending the operations. Let $\vartheta_{n}: \mathcal{M} \rightarrow \mathbb{U}$ be as in Lemma 2.3. To each $\xi \in \hat{\Xi}$ we have the associated $d_{\xi} \in \mathcal{M}$ defined by $d_{\xi}(n, m)=\|n-m\|_{\xi}$, and the map $\xi \mapsto d_{\xi}$ is clearly Borel. It is then straightforward to define $\mathbb{X}^{\xi}=\left(X^{\xi}, F_{0}^{\xi}, F_{+}^{\xi}, F^{\xi}, F_{*}^{\xi},\left(F_{q}^{\xi}\right)_{q \in \mathbb{Q}(i)}\right) \in M_{C^{*}}$ directly from $d_{\xi}$ and the $\vartheta_{n}$ so that $\mathbb{X}^{\xi}$ is isomorphic to $C^{*}(\xi)$, setting $F_{0}^{\xi}=\left\{\vartheta_{0_{\xi}}\left(d_{\xi}\right)\right\}$,

$$
F_{+}^{\xi}=\left\{\left(\vartheta_{n}\left(d_{\xi}\right), \vartheta_{m}\left(d_{\xi}\right), \vartheta_{k}\left(d_{\xi}\right)\right): n+\xi m=k\right\},
$$

and defining $F^{\xi}, F_{*}^{\xi},\left(F_{q}^{\xi}\right)_{q \in \mathbb{Q}(i)}$ analogously. The map $\xi \mapsto \mathbb{X}^{\xi}$ is then Borel by the Kuratowski-Ryll-Nardzewski theorem.

For the converse direction, recall from [7, Section 2.4] that $\Xi \subseteq \mathbb{R}^{\mathbb{N}}$ consists of all real sequences $\eta$ such that for some $\mathrm{C}^{*}$-algebra $A$ and some $y=\left(y_{n}\right)_{n \in \mathbb{N}}$ which is dense in $A$, we have that $\eta_{n}=\left\|\mathfrak{p}_{n}(y)\right\|_{A}$, where $\left(\mathfrak{p}_{n}\right)_{n \in \mathbb{N}}$ enumerates the non-commutative $\mathbb{Q}(i)$-*-polynomials. Let $f_{n}: F(\mathbb{U}) \backslash\{\varnothing\} \rightarrow \mathbb{U}, n \in \mathbb{N}$, be Borel functions provided by the Kuratowski-Ryll-Nardzewski theorem such that $\left\{f_{n}(X): n \in \mathbb{N}\right\}$ is a dense subset of $X$, for all $X \in F(\mathbb{U}) \backslash\{\varnothing\}$. For $\mathbb{X} \in M_{C^{*}}$, set $\eta_{n}^{\mathbb{X}}=\delta\left(\mathfrak{p}_{n}\left(\left(f_{n}(\mathbb{X})_{n \in \mathbb{N}}\right), 0_{\mathbb{X}}\right)\right.$ where $F_{0}^{\mathbb{X}}=\left\{0_{\mathbb{X}}\right\}$, and $\mathfrak{p}_{n}\left(\left(f_{n}(\mathbb{X})\right)\right.$ is the evaluation of $\mathfrak{p}_{n}$ at $y=\left(f_{n}(X)\right)$ in the $\mathrm{C}^{*}$-algebra coded by $\mathbb{X}$. It is easily seen that $\mathbb{X} \mapsto \eta^{\mathbb{X}}$ is Borel and $\mathbb{X}$ and $\eta^{\mathbb{X}}$ encode isomorphic $\mathrm{C}^{*}$-algebras.

3.2. Banach spaces and Banach algebras. A separable (real or complex) Banach space $E$ can be cast as a Polish structure $\left(E, F_{0}, F_{+},\left(F_{q}\right)_{q \in \mathbb{K}}\right)$, where $\mathbb{K}=\mathbb{Q}$ or $\mathbb{K}=\mathbb{Q}(i)$, in the signature $\mathcal{L}_{\mathrm{B}}=(1,3,2,2, \ldots)$ in analogy with the above parametrization of $\mathrm{C}^{*}$-algebras (omitting multiplication and involution). The subset $M_{\mathrm{B}}(\mathbb{U})$ of $M\left(\mathcal{L}_{\mathrm{B}}, \mathbb{U}\right)$ corresponding to Banach spaces is easily seen to be Borel, too. By an argument similar to that in Section 3.1, one sees that this parametrization is equivalent to the standard parametrization of Banach spaces as closed subspaces of $C([0,1])$ (see, e.g., [8] or [14]).

In a similar vein, letting $\mathcal{L}_{\mathrm{BA}}=(1,3,3,2,2, \ldots)$ (which happens to coincide with $\mathcal{L}_{\mathrm{C}^{*}}$ ), we can parametrize separable Banach algebras, as well as separable involutive Banach algebras, by appropriate Borel subsets of $M\left(\mathcal{L}_{\mathrm{BA}}\right)$.

In all cases, Theorem 2.2 implies that the isometric isomorphism relation is below a group action.

3.3. Operator spaces and operator systems. To handle operator spaces and operator systems we use a framework slightly more general than that of Polish structures. Formally, this could be handled by introducing multi-sorted Polish structures, though here we make do with an ad hoc approach.

For metric spaces $\left(X, d_{X}\right)$ and $\left(Y, d_{Y}\right)$, let $U C(X, Y)$ denote the set of uniformly continuous functions $f: X \rightarrow Y$. Identifying each $f \in U C(X, Y)$ with $\operatorname{graph}(f) \subseteq$ $X \times Y$, it is easily checked that $U C(X, Y)$ forms a Borel subset of $F(X \times Y)$.

Let $\mathbb{U}_{n}, n \in \mathbb{N}$, be a sequence of disjoint copies of the Urysohn metric space, with $\mathbb{U}_{1}=\mathbb{U}$. Identify $M_{n}(\mathbb{U})$ with $\mathbb{U}^{n^{2}}$, and give this the supremum metric. We define $\mathbb{O S}$ to be the set of sequences $\mathbb{E}=\left(E_{n}, f_{n}\right)_{n \in \mathbb{N}}$ such that:

(1) $\left(E_{n}, f_{n}\right) \in M_{\mathrm{B}}\left(\mathbb{U}_{n}\right) \times U C\left(M_{n}(\mathbb{U}), \mathbb{U}_{n}\right)$;

(2) $f_{n}\left(M_{n}\left(E_{1}\right)\right)=\operatorname{dom}\left(E_{n}\right)$; 
(3) $f_{n}\left\lceil M_{n}\left(E_{1}\right)\right.$ is linear;

(4) for all $A, B \in M_{n}(\mathbb{C})$ and all $X \in M_{n}\left(E_{1}\right)$ we have

$$
\left\|f_{n}(A X B)\right\|_{E_{n}} \leq\|A\|\left\|f_{n}(X)\right\|_{E_{n}}\|B\| ;
$$

(5) for all $m, n \in \mathbb{N}$ and all $X \in M_{n}\left(E_{1}\right), Y \in M_{m}\left(E_{1}\right)$,

$$
\|X \oplus Y\|_{E_{n+m}}=\max \left\{\|X\|_{E_{n}},\|Y\|_{E_{m}} \|\right\} .
$$

Once again, $\mathbb{O S}$ forms a Borel set. Each $\mathbb{E} \in \mathbb{O S}$ codes an $L^{\infty}$-matrix-normed space, whence by Ruan's theorem (e.g., [15, Theorem 13.4]), OS parametrizes the class of separable operator spaces. Defining $\mathbb{E} \simeq \mathbb{O S} \mathbb{F}$ if and only if $\mathbb{E}$ and $\mathbb{F}$ are completely isometrically isomorphic, one checks that $\simeq \mathbb{O S}$ is analytic, and so $\mathbb{O S}$ provides a good standard Borel parametrization of separable operator spaces. Also, write $\mathbb{E} \simeq_{n}^{\mathbb{S}} \mathbb{F}$ if and only $\mathbb{E}$ and $\mathbb{F}$ are $n$-isometric.

The group Iso $(\mathbb{U}) \times \operatorname{Iso}\left(\mathbb{U}_{n}\right)$ acts in a Borel way on $U C\left(M_{n}(\mathbb{U}), \mathbb{U}_{n}\right)$ by

$$
((\sigma, \tau) \cdot f)\left(x_{i j}\right)=\tau\left(f\left(\sigma^{-1}\left(x_{i j}\right)\right) .\right.
$$

Thus we obtain a Borel action of $\prod_{n} \operatorname{Iso}\left(\mathbb{U}_{n}\right)$ on $\mathbb{O S}$ by

$$
\left(\sigma_{n}\right) \cdot\left(E_{n}, f_{n}\right)=\left(\sigma_{n} \cdot E_{n},\left(\sigma_{1}, \sigma_{n}\right) \cdot f_{n}\right),
$$

and we write $\mathbb{E} \equiv \mathbb{O S} \mathbb{F}$ if and only there is $\left(\sigma_{n}\right)$ such that $\left(\sigma_{n}\right) \cdot \mathbb{E}=\mathbb{F}$. Arguing as we did in the proof of Theorem 2.2, we obtain that $\simeq^{\mathbb{O S}} \leq_{\mathrm{B}} \equiv{ }^{\mathbb{O S}}$, which proves that $\simeq^{\mathbb{O S}}$ is below a group action.

If we only consider the action of $\prod_{j \leq n} \operatorname{Iso}\left(\mathbb{U}_{n}\right)$ on $\mathbb{O S}$, and denote by $\equiv_{n}^{\mathbb{O S}}$ the induced orbit equivalence relation, the argument from Theorem 2.2 gives that $\simeq_{n}^{\mathbb{O S}}$ $\leq_{\mathrm{B}} \equiv_{n}^{\mathbb{O S}}$, thus showing $\simeq_{n}^{\mathbb{O S}}$ is below a group action. This establishes Theorem 1.1.(2).

Theorem 1.1.(3) follows immediately from (2) after a standard parametrization of operator systems is obtained by adding the adjoint operation to the structures parametrized by $\mathbb{O S}$.

By adding a constant for the unit of a unital operator system to the language one sees that the relation of unital complete isometry between operator systems is below a group action. It remains to prove that $n$-order isomorphism of operator systems is below a group action. The case that $n \geq 2$ is a consequence of the fact that unital maps between operator systems that are $n$-positive for $n \geq 2$ are necessarily $\lfloor n / 2\rfloor$ contractive (this easily follows from the proof of the case that $n=2$ given in $[15$, Proposition 3.2]). Aside from this, the assertion follows directly from Theorem 2.2. Indeed, a Polish structure coding an operator system $S$ consists of $(S,+,\|\cdot\|)$ and for each $2 \leq k \in \mathbb{N}$ the closed subset $P_{k}(S)$ of $S^{k^{2}}$ consisting of all positive elements of $M_{k}(S)$. By the above, a unital $n$-order isomorphism between two unital operator systems $S$ and $S^{\prime}$ is implemented by an isometry that sends $P_{k}(S)$ onto $P_{k}\left(S^{\prime}\right)$ for all $k \leq n$ and the case $n \geq 2$ of Theorem 1.1 (4) follows.

Let us now prove the case $n=1$ of Theorem 1.1 (4), and at the same time give an alternative proof of the general case. Recall that the numerical radius of an operator $X$ is defined by

$$
\||X|\| \mid=\sup \{|s(X)|: s \text { is a state }\}
$$


and that $\||| X|\|\leq\| X\|\leq 2 \mid\| X\|\|$ (see, e.g., [16, Proposition 3.2.25]). By an argument similar to the above, it will suffice to prove that a linear map is a unital order isomorphism if and only if it is a linear unital isometry in this norm. If $\phi: S \rightarrow T$ is a unital positive map and $s: T \rightarrow \mathbb{C}$ is a state, then $s \circ \phi: S \rightarrow \mathbb{C}$ is a state. Therefore, $\||\phi(X)\|\| \leq\||X|\|$. Thus, if $\phi$ has a positive inverse then it is a $\|| \cdot|| \mid$-isometry. The conclusion now follows by the arguments used above.

Note that one can prove (2) and (3) of Theorem 1.1 by using a proof similar to the proof of (4) and the remark about adding predicates made after the definition of a Polish structure.

We do not know whether either of the relations non-unital $n$-order isomorphism or non-unital complete order isomorphism of operator systems is below a group action.

3.4. A remark about models of the logic for metric structures. The isomorphism relation for countable structures, in the sense of [10], is given by a continuous $S_{\infty}$-action. The group Iso(U) plays an analogous role for separable models of logic for metric structures. Such models consist of an underlying Polish space $X$, countably many functions $f_{n}: X^{n} \rightarrow X$, and countably many functions $g_{n}: X^{n} \rightarrow \mathbb{R}$ (relations). (Assuming there are infinitely many functions and that the $n$th function has $X^{n}$ as its domain is clearly not a loss of generality.) These functions are required to have a prescribed modulus of uniform continuity (see [1]), but we shall ignore this since it is not important for our present purposes. To a fixed model $\mathcal{X}=\left(X, f_{n}, g_{n}: n \in \mathbb{N}\right)$, associate a Polish structure $\mathbb{X}=\left(X,\left(F_{n}\right)_{n \in \mathbb{N}}\right)$ where $F_{n}, n \in \mathbb{N}$, enumerate the graphs of the $f_{n}$, as well as all the sets $\left\{\bar{x} \in X^{n}: g_{n}(x) \geq q_{n}\right\}$ where $\left(q_{n}\right)_{n \in \mathbb{N}}$ is a fixed enumeration of the rationals. This map is Borel (between the appropriate spaces) and $\mathcal{X}$ is isomorphic to $\mathcal{Y}$ if and only if $\mathbb{X} \simeq \mathbb{Y}$. Also, the map that sends an element of $\mathcal{M}$ to a metric structure is Borel.

The following statement is proved by a straightforward recursion analogous to the case of classical logic.

Lemma 3.1. If $T$ is a theory in a countable language in the logic of metric structures then the set of all $\mathbb{X} \in \mathcal{M}$ that encode a model of $T$ is Borel.

Combining this with Theorem 2.2, we obtain:

Theorem 3.2. If $T$ is a theory of the logic of metric structures in a separable language, then the isometry relation of models of $T$ is Borel reducible to an orbit equivalence relation of $\mathrm{Iso}(\mathbb{U})$.

\section{Concluding remarks}

(1) Aaron Tikuisis has pointed out that the above approach, using Polish structures, also can be used to provide a new proof that the isomorphism relation for von Neumann algebras with separable predual is below a group action. Previously, in [18], it was shown that isomorphism of separably acting von Neumann algebras is below an action of the unitary group of $l_{2}$, using a completely different line of argument. It is at present not known if the latter provides a sharper upper bound on complexity than the former.

(2) In [7] it was proved that separable unital AI algebras are not CCS, and that their isomorphism relation is below an action of $\operatorname{Aut}\left(\mathcal{O}_{2}\right)$. However, we do not at present 
know if the complexity of the classification problem increases as one passes from nuclear to exact $\mathrm{C}^{*}$-algebras, or from exact to arbitrary $\mathrm{C}^{*}$-algebras. In particular, we do not know the answer to: ${ }^{2}$

Question 4.1. Is the isomorphism relation for separable $C^{*}$-algebras strictly more complicated, as measured by $\leq_{\mathrm{B}}$, than that of nuclear separable $C^{*}$-algebras?

We may also ask if the upper bound on complexity provided by Theorem 1.1.(1) is actually optimal. By [9], the Borel actions of Iso(UU) realize the maximal complexity of equivalence relations induced by Polish group actions.

Question 4.2. Is the isomorphism relation for separable $C^{*}$-algebras maximal among equivalence relations induced by a Polish group action?

\section{Acknowledgments}

The main result of this paper was proved in the aftermath of the June 2012 BIRS workshop on applications of descriptive set theory to functional analysis. We would like to thank the staff at BIRS for providing an inspiring atmosphere.

G.A.E. and I.F. were partially supported by NSERC. V.P. was partially supported by NSF grant DMS-1101231. C.R. was partially supported by NSF grants 1201295 and 0901405. A.T. was supported in part by grant no. 10-082689 from the Danish Council for Independent Research.

\section{Appendix}

We prove the generalization of the homogeneous selection principle, [7, Lemmas 6.2 and 6.3], which is used to prove Lemma 2.3 above. For a subset $A \subseteq X \times Y$ of a Cartesian product and $x \in X$, define $A_{x}=\{y \in Y:(x, y) \in A\}$. A function $f: \operatorname{proj}_{X}(A) \rightarrow Y$ is a uniformization of $A$ if $(x, f(x)) \in A$ for all $x \in \operatorname{proj}_{X}(A)$.

Theorem A. Let $X, Y$ be Polish spaces, $d_{Y}$ a complete compatible metric on $Y$, and let $A \subseteq X \times Y$ be a $G_{\delta}$ set.

(1) If for some (any) sequence $\left(y_{n}\right)_{n \in \mathbb{N}}$ dense in $Y$ the set

$$
R=\left\{(x, n, \varepsilon) \in X \times \mathbb{N} \times \mathbb{Q}_{+}:\left(\exists y \in A_{x}\right) d_{Y}\left(y, y_{n}\right)<\varepsilon\right\}
$$

is Borel, then $\operatorname{proj}_{X}(A)$ is Borel and $A$ admits a Borel uniformization.

(2) If $G$ is a Polish group acting continuously on $Y$ by Borel automorphisms such that $A_{x}$ is $G$-invariant for all $x \in X$, and every $G$-orbit of a point $y \in A_{x}$ is dense in $A_{x}$, then $R$ as defined in (1) is Borel, and so $A$ admits a Borel uniformization.

Proof. We may assume that $d_{Y}$ is bounded by $\frac{1}{2}$. Clearly $\operatorname{proj}_{X}(A)=\{x \in X$ : $(\exists n)(x, n, 1) \in R\}$, and so this set is Borel. To construct the uniformization, fix open sets $U_{n} \subseteq X \times Y$ such that $\bigcap_{n \in \mathbb{N}} U_{n}=A$, and fix an enumeration $\left(q_{n}\right)_{n \in \mathbb{N}}$ of $\mathbb{Q}_{+}$. Let $B(y, \varepsilon)$ denote the open $d_{Y}$-ball of radius $\varepsilon$ around $y \in Y$. We recursively define

\footnotetext{
${ }^{2} \mathrm{~A}$ negative answer to Question 4.1 and a positive answer to Question 4.2 have been given in [17].
} 
Borel maps $x \mapsto n_{i}(x) \in \mathbb{N}$ and $x \mapsto \varepsilon_{i}(x) \in \mathbb{Q}_{+}, i \in \mathbb{N}$, on $\operatorname{proj}_{X}(A)$, satisfying:

(1) $n_{i}(x)=0, \varepsilon_{0}(x)=1$;

(2) $\varepsilon_{i}(x) \leq \frac{1}{2^{i}}$

(3) $B\left(y_{n_{i}(x)}, \varepsilon_{i}(x)\right) \subseteq\left(U_{i}\right)_{x}$;

(4) $\left(x, n_{i}(x), \varepsilon_{i}(x)\right) \in R$;

(5) $\overline{B\left(y_{n_{i+1}(x)}, \varepsilon_{i+1}\right)} \subseteq B\left(y_{n_{i}(x)}, \varepsilon_{i}(x)\right)$.

If this can be done then $f(x)=\lim _{i \rightarrow \infty} y_{n_{i}(x)}$ is the desired Borel uniformization. Suppose that $n_{i}(x)$ and $\varepsilon_{i}(x)$ have been defined for $i \leq k$. Set

$$
z \in B\left(y_{n_{k}(x)}, \varepsilon_{k}(x)\right) \cap A_{x}
$$

and let $0<\delta \leq 2^{k+1}$ be such that $2 \delta+d_{Y}\left(z, y_{n_{k}(x)}\right)<\varepsilon_{k}(x)$ and $B(z, 2 \delta) \subseteq U_{k+1}$. If $d\left(y_{n}, z\right)<\delta$, then $\overline{B\left(y_{n}, \delta\right)} \subseteq B\left(y_{n_{k}(x)}, \varepsilon_{k}(x)\right), B\left(y_{n}, \delta\right) \cap A_{x} \neq \varnothing$, and $B\left(y_{n}, \delta\right) \subseteq$ $U_{k+1}$. This shows that (1)-(5) above can be satisfied, and so we can define $n_{k+1}(x)$ and $\varepsilon_{k+1}(x)=q_{n}$, where $n_{k+1}(x)$ and $n$ are least possible satisfying (1)-(5) above. Since each of the requirements (1)-(5) is Borel, the maps $x \mapsto n_{k+1}(x)$ and $x \mapsto \varepsilon_{k+1}(x)$ are Borel, as required.

It is clear from the definition that $R$ is analytic. Since

$$
R=\left\{(x, n, \varepsilon):\left(\forall y \in A_{x}\right)\left(\exists g \in G_{0}\right) d\left(g \cdot y, y_{n}\right)<\varepsilon\right\}
$$

for a countable dense $G_{0} \subseteq G$ it is also coanalytic, whence $R$ is Borel.

\section{References}

[1] I. Ben Yaacov, A. Berenstein, C. Henson and A. Usvyatsov, Model theory for metric structures, in 'Model theory with applications to algebra and analysis', (Z. Chatzidakis et al., eds.), Vol. II, number 350 in London Math. Soc. Lecture Notes Series, 315-427, Cambridge University Press, 2008.

[2] G. A. Elliott and A. S. Toms, Regularity properties in the classification program for separable amenable $C^{*}$-algebras, Bull. Amer. Math. Soc. 45(2) (2008), 229-245.

[3] G. A. Elliott, Towards a theory of classification, Adv. Math. 223(1) (2010), 30-48.

[4] I. Farah, A dichotomy for the Mackey Borel structure, Proc. 11th Asian Logic Conf., 86-93, World Sci. Publ., Hackensack, NJ, 2012.

[5] I. Farah, B. Hart and D. Sherman, Model theory of operator algebras II: Model theory, Israel J. Math. (to appear).

[6] I. Farah, A. S. Toms and A. Törnquist, The descriptive set theory of $C^{*}$-algebra invariants, Int. Math. Res. Notices 22 (2013) 5196-5226.

[7] - Turbulence, orbit equivalence, and the classification of nuclear $C^{*}$-algebras, Journal fr die reine und angewandte Mathematik (Crelle's Journal), 688 (2014) 101-146.

[8] V. Ferenczi, A. Louveau and C. Rosendal, The complexity of classifying separable Banach spaces up to isomorphism, J. Lond. Math. Soc. (2) 79(2) (2009), 323-345.

[9] S. Gao and A. S. Kechris, On the classification of Polish metric spaces up to isometry, Mem. Amer. Math. Soc. 161(766) (2003), viii+78.

[10] G. Hjorth, Classification and orbit equivalence relations, Vol. 75 of Mathematical Surveys and Monographs, American Mathematical Society (2000).

[11] M. Katětov, On universal metric spaces, General topology and its relations to modern analysis and algebra, VI (Prague, 1986), Vol. 16 (Res. Exp. Math.), 323-330, Heldermann, Berlin, 1988.

[12] A. Kechris, Classical descriptive set theory, Vol. 156 of Graduate Texts in Mathematics, Springer, 1995.

[13] D. Kerr, H. Li and M. Pichot, Turbulence, representations, and trace-preserving actions, Proc. Lond. Math. Soc. (3) 100(2) (2010), 459-484.

[14] J. Melleray, Computing the complexity of the relation of isometry between separable Banach spaces, MLQ Math. Log. Q. 53(2) (2007), 128-131. 
[15] V. Paulsen, Completely bounded maps and operator algebras, Vol. 78 of Cambridge Studies in Advanced Mathematics, Cambridge University Press, Cambridge, 2002, ISBN 0-521-81669-6.

[16] G. K. Pedersen, Analysis now, Vol. 118 (Graduate Texts in Mathematics), Springer-Verlag, New York, 1989.

[17] M. Sabok, Completeness of the isomorphism problem for separable $C^{*}$-algebras, 2013, arXiv:1306.1049.

[18] R. Sasyk and A. Törnquist, The classification problem for von Neumann factors, J. Funct. Anal. 256(8) (2009), 2710-2724.

[19] — Turbulence and Araki-Woods factors, J. Funct. Anal. 259(9) (2010), 2238-2252.

Department of Mathematics, University of Toronto, Toronto, Ontario M5S 2E4, CANADA

E-mail address: elliott@math.toronto.edu

Department of Mathematics and Statistics, York University, 4700 Keele St., Toronto, ONTARIO M3J 1P3, CANADA

E-mail address: ifarah@mathstat.yorku.ca

Department of Mathematics, University of Houston, Houston, TX 77204-3476, USA

E-mail address: vern@math.uh.edu

Department of Mathematics, Statistics, and Computer Science (M/C 249), University of Illinois at Chicago, 851 S. Morgan St., Chicago, IL 60607-7045, USA

E-mail address: rosendal.math@gmail.com

Department of Mathematics, Purdue University, 150 N. University St., West Lafayette, IN 47906, USA

E-mail address: atoms@purdue.edu

Department of Mathematical Sciences, University of Copenhagen, Universitetsparken 5, 2100 Copenhagen, Denmark

E-mail address: asgert@math.ku.dk 